\title{
SEMI- $p$-FUNCTIONS
}

BY

\section{J. F. C. KINGMAN}

ABSTRACT. A generalisation of the theory of $p$-functions which applies, for instance, to the diagonal elements of one-parameter semigroups of infinite matrices which satisfy no boundedness condition.

1. Semigroups of positive matrices. The classical theory of Markov chains [1] with a continuous time parameter and a countable infinity of states (here labelled by the positive integers) is, in large measure, the study of systems of functions $p_{i j}(i, j=1,2,3, \cdots)$ of a positive real variable, satisfying the conditions

$$
\begin{aligned}
p_{i j}(t) & \geq 0, \\
\sum_{j=1}^{\infty} p_{i j}(t) & =1, \\
p_{i j}(s+t) & =\sum_{k=1}^{\infty} p_{i k}(s) p_{k j}(t), \\
\lim _{t \rightarrow 0} p_{i j}(t) & =\delta_{i i} .
\end{aligned}
$$

These conditions are known to entail very special properties of the functions $p_{i j}$, and a question which is implicit for example in Kolmogorov's 1951 paper

[14] is that of characterising these functions. This problem was solved in [12], the solution turning upon the concept of a p-function introduced in [8].

For any real-valued $p$ of a positive real variable, define the quantities $F\left(t_{1}, t_{2}, \cdots, t_{n} ; p\right)$ and $G\left(t_{1}, t_{2}, \cdots, t_{n} ; p\right)$ for $n \geq 1$ and

$$
0=t_{0}<t_{1}<t_{2}<\cdots<t_{n}
$$

by

(6) $\quad F\left(t_{1}, t_{2}, \cdots, t_{n} ; p\right)=\sum_{r=0}^{n-1}(-1)^{r} \sum_{0<a_{1}<\cdots<a_{r}<n} p\left(t_{a_{1}}\right) p\left(t_{a_{2}}-t_{\alpha_{1}}\right) \cdots p\left(t_{n}-t_{a_{r}}\right)$ and

$$
G\left(t_{1}, t_{2}, \cdots, t_{n} ; p\right)=1-\sum_{m=1}^{n} F\left(t_{1}, t_{2}, \cdots, t_{m} ; p\right)
$$

Received by the editors October 26, 1971.

AMS (MOS) subject classifications (1970). Primary 26A51. 
Then $p$ is said to be a $p$-function if, for all $n \geq 1$ and all $t_{r}$ satisfying (5),

$$
F\left(t_{1}, t_{2}, \cdots, t_{n} ; p\right) \geq 0, \quad G\left(t_{1}, t_{2}, \cdots, t_{n} ; p\right) \geq 0 .
$$

The point of this definition is that a simple calculation shows that, if the functions $p_{i j}$ satisfy (1)-(3), then

(9) $F\left(t_{1}, t_{2}, \cdots, t_{n} ; p_{11}\right)=\sum_{k_{1}, k_{2}, \cdots, k_{n-1}=2}^{\infty} p_{1 k_{1}}\left(t_{1}\right) p_{k_{1} k_{2}}\left(t_{2}-t_{1}\right) \cdots p_{k_{n-1} 1}\left(t_{n}-t_{n-1}\right)$,

and that

(10) $G\left(t_{1}, t_{2}, \cdots, t_{n} ; p_{11}\right)=\sum_{k_{1}, k_{2}} \sum_{\cdots, k_{n}=2}^{\infty} p_{1 k_{1}}\left(t_{1}\right) p_{k_{1} k_{2}}\left(t_{2}-t_{1}\right) \cdots p_{k_{n-1} k_{n}}\left(t_{n}-t_{n-1}\right)$.

Since the right-hand sides of (9) and (10) are nonnegative, $p_{11}$ (and similarly $\left.p_{i i}\right)$ is a $p$-function.

A $p$-function $p$ is standard if

$$
\lim _{t \rightarrow 0} p(t)=1
$$

(so that $p_{11}$ is standard if (4) holds) and the theory of standard $p$-functions is developed in [9] and [10]. The basic theorem is that a standard $p$-function is continuous, and that its Laplace transform,

$$
\hat{p}(\theta)=\int_{0}^{\infty} p(t) e^{-\theta t} d t
$$

which exists for $\theta>0$, can be written in the form

$$
\hat{p}(\theta)=\left\{\theta+\int_{(0, \infty]}\left(1-e^{-\theta x}\right) \mu(d x)\right\}^{-1},
$$

where $\mu$ is a unique (positive Borel) measure on $(0, \infty]$ with

$$
\int_{(0, \infty]}\left(1-e^{-x}\right) \mu(d x)<\infty \text {. }
$$

Conversely, if $\mu$ is any measure on $(0, \infty]$ satisfying (14), there is a unique continuous function $p$ satisfying (13), and $p$ is a standard $p$-function.

It is not true that every standard $p$-function can be written in the form $p_{11}$ for some system of functions $p_{i j}$ satisfying (1)-(4); [12] gives the necessary and sufficient conditions on $\mu$ for this to be possible.

Of the conditions (1)-(4), (1) and (3) assert that the infinite matrices $P_{t}=$ $\left(p_{i j}(t)\right)$ are positive and form a one-parameter semigroup under matrix multiplication. Condition (4) is just a way of eliminating those semigroups whose dependence on $t$ is pathological (cf. [1]). But (2), though it emerges naturally from the Markovian theory, is, from a more general point of view, somewhat arbitrary, and even within probability theory there are situations (for instance in the theory of multitype branching processes) in which (2) is absent. It is therefore of great 
interest that Jurkat $([4],[5])$ has shown that many properties of the solutions of (1)-(4) persist even if (2) is dropped. More recent work by Cornish [2] supports the view that (2) is largely irrelevant.

The derivation of equation (9) depends only on (3), but that of (10) uses (2) as well. Thus if $\left(p_{i j}\right)$ satisfies (1) and (3), it is true that

$$
F\left(t_{1}, t_{2}, \cdots, t_{n} ; p_{11}\right) \geq 0
$$

but not necessarily that $G\left(t_{1}, t_{2}, \cdots, t_{n} ; p_{11}\right) \geq 0$. Thus the problem arises of studying the properties of functions which satisfy one-half of the $p$-function inequalities (8) but not the other. These will be called semi-p-functions, and will be shown to have many of the properties associated with $p$-functions.

2. Generalised renewal sequences. Let $u=\left(u_{n} ; n \geq 0\right)$ be any sequence of real numbers with $u_{0}=1$, and define $f_{n}$ to be the coefficient of $z^{n}$ in the formal power series

$$
\sum_{n=1}^{\infty} f_{n} z^{n}=1-\left\{\sum_{n=0}^{\infty} u_{n} z^{n}\right\}^{-1}
$$

Then $f_{n}$ is a polynomial $\phi_{n}(u)$ in $u_{1}, u_{2}, \cdots, u_{n}$, and similarly $u_{n}$ is a polynomial in $f_{1}, f_{2}, \cdots, f_{n}$. The relation between the two sequences may be equivalently expressed by the equations

$$
u_{0}=1, \quad u_{n}=\sum_{r=1}^{n} f_{r} u_{n-r} \quad(n \geq 1) .
$$

The sequence $u$ is called a renewal sequence [11] if

$$
\phi_{n}(u) \geq 0 \quad(n \geq 1)
$$

and

$$
\sum_{n=1}^{\infty} \phi_{n}(u) \leq 1
$$

The the ory of semi- $p$-functions involves sequences which satisfy (18) but not necessarily (19); these will be called generalised renewal sequences. The following simple results have important consequences.

Proposition 1. A generalised renewal sequence $u$ is a renewal sequence if and only if

$$
u_{n} \leq 1
$$

for all $n$.

Proof. It is well known (and follows from (17) by induction) that a renewal sequence satisfies $0 \leq u_{n} \leq 1$, so that the necessity of $(20)$ is evident. Conversely, suppose that the generalised renewal sequence $u$ satisfies (20), and 
write $f_{n}=\phi_{n}(u) \geq 0$. By induction on (17), $u_{n} \geq 0$, and so $u_{n} \geq f_{n}$. Hence $0 \leq$ $f_{n} \leq u_{n} \leq 1$, and the two power series in (16) can be regarded as power series in a real variable $z$ which converge in $|z|<1$. Then (16) implies that $\sum_{n=1}^{\infty} f_{n} z^{n} \leq 1$ $(0 \leq z<1)$, and letting $z \rightarrow 1$ we have $\sum_{n=1}^{\infty} f_{n} \leq 1$, and the proof is complete.

If $u$ is a sequence with $u_{0}=1$, and $b$ is any positive number, then the sequence $v$ defined by

$$
v_{n}=u_{n} b^{n}
$$

has

$$
\sum_{n=1}^{\infty} \phi_{n}(v) z^{n}=1-\left\{\sum_{n=0}^{\infty} u_{n} b^{n} z^{n}\right\}^{-1}=\sum_{n=1}^{\infty} \phi_{n}(u)(b z)^{n}
$$

so that

$$
\phi_{n}(v)=\phi_{n}(u) b^{n}
$$

Hence $v$ is a generalised renewal sequence if and only if $u$ is.

In particular, suppose that $u$ is a generalised renewal sequence satisfying

$$
u_{n} \leq c^{n}
$$

for some positive c. Taking $b=c^{-1}$ in (21) and applying Proposition 1 to $v$, we see that

$$
u_{n}=v_{n} c^{n}
$$

where $v$ is a renewal sequence. Thus generalised renewal sequences satisfying (23) are not essentially different from renewal sequences. New problems only arise with generalised renewal sequences which increase so fast that (23) is not satisfied for any $c$, however large. The next result shows that, even then, the finite segments of such sequences present no new phenomena.

Proposition 2. A sequence $\left(u_{n}\right)$ with $u_{0}=1$ is a generalised renewal sequence if and only if, for each $N \geq 1$, there exist a renewal sequence $\left(v_{n}\right)$ and a positive number $c$ such that

$$
u_{n}=v_{n} c^{n} \quad(n=1,2, \cdots, N) .
$$

Proof. The condition is sufficient, since (25) implies (using (22) and the fact that $\phi_{n}(u)$ only depends on $\left.u_{1}, u_{2}, \cdots, u_{n}\right)$ that $\phi_{n}(u)=\phi_{n}(v)_{c}^{n} \geq 0$ for $n \leq N$, and $N$ is arbitrary.

Conversely, suppose that $u$ is a generalised renewal sequence, and fix $N \geq 1$. Choose $c$ so large that 


$$
\sum_{n=1}^{N} \phi_{n}(u)_{c}^{-n} \leq 1
$$

and let $\left(v_{n}\right)$ be the renewal sequence with

$$
\begin{aligned}
\phi_{n}(v) & =\phi_{n}(u)_{c^{-n}} & & (1 \leq n \leq N), \\
& =0 & & (N<n) .
\end{aligned}
$$

Then $\bar{u}_{n}=v_{n} c^{n}$ defines a sequence with $\phi_{n}(\bar{u})=\phi_{n}(v)_{c^{n}}=\phi_{n}(u)(n \leq N)$ and therefore $\bar{u}_{n}=u_{n}(n \leq N)$. This establishes (25) and completes the proof.

It should be noted that, although in Proposition 2 it is clearly necessary that $c$ be chosen so large that

$$
u_{n} \leq c^{n} \quad(n=1,2, \cdots, N)
$$

this is not always sufficient. For example, the sequence

$$
u_{n}=\frac{2}{3}\left\{\left(\frac{4}{3}\right)^{n / 2}+\frac{1}{2}(-1)^{n}\left(\frac{1}{3}\right)^{n / 2}\right\}
$$

is a generalised renewal sequence since $\phi_{1}(u)=1 / 3^{1 / 2}, \phi_{2}(u)=2 / 3, \phi_{n}(u)=0$ $(n \geq 3)$, and it satisfies (27) with $c=1$ and $N=2$ because $u_{1}=1 / 3^{1 / 2}, u_{2}=1$. But there is no renewal sequence $v$ satisfying (25) with $c=1, N=2$, since such a sequence would have

$$
\sum_{n=1}^{\infty} \phi_{n}(v) \geq \phi_{1}(v)+\phi_{2}(v)=\phi_{1}(u)+\phi_{2}(u)>1 .
$$

In fact, the smallest value of $c$ for this sequence and $N=2$ is $c=2 / 3^{1 / 2}$ (and this example is the worst when $N=2$ ). Without this phenomenon, the arguments of $\$ 4$ would be somewhat easier.

3. Some examples. It has been seen that generalised renewal sequences only differ significantly from ordinary renewal sequences when they grow faster than any geometric progression. A generalised renewal sequence will therefore be described as tame if it satisfies (23) for some $c$, and as wild otherwise. Then Proposition 2 asserts that every wild sequence agrees on any finite interval with a tame sequence. The next result exhibits a large family of generalised renewal sequences, some of which are tame and others wild.

Proposition 3. Let $u=\left(u_{n} ; n=0,1,2, \ldots\right)$ be a sequence of positive numbers such that $u_{n}=1$ and the sequence

$$
r_{n}=u_{n+1} / u_{n}
$$

is nondecreasing. Then $u$ is a generalised renewal sequence, and is tame if and only if

$$
r=\lim _{n \rightarrow \infty} r_{n}
$$

is finite. 
Proof. For any $N \geq 1$, define

$$
\begin{array}{rlrl}
c=r_{N}, \quad v_{n} & =u_{n} c^{-n} & & (n \leq N), \\
& =u_{N} c^{-N} \quad(n>N) .
\end{array}
$$

Then $0<v_{n} \leq 1=v_{0}$ and $v_{n+1} v_{n-1} \geq v_{n}^{2}$ for all $n$. Thus ([11], [6]) $v$ is a 'Kaluza sequence' and is hence a renewal sequence. Proposition 2 then shows that $u$ is a generalised renewal sequence. Moreover, it is clear that (23) holds if and only if $c \geq r$, so that $u$ is tame if and only if $r$ is finite.

The wild generalised renewal sequences whose existence is assured by the proposition may be used to construct examples of matrix semigroups satisfying (1), (3) and (4), which may be added to the interesting collection produced by Cornish [2].

Proposition 4. Let $u$ be any generalised renewal sequence such that the series

$$
p(t)=\sum_{n=0}^{\infty} u_{n} t^{n} e^{-t} / n !
$$

converges for all $t>0$. Then there exists a collection of functions $p_{i j}(i, j=$ $1,2, \ldots)$ such that

$$
p_{11}(t)=p(t)
$$

In particular, if $u$ is a sequence of the type envisaged in Proposition 3, where the nondecreasing sequence $\left(r_{n}\right)$ satisfies

$$
\lim _{n \rightarrow \infty} r_{n}=\infty, \quad \lim _{n \rightarrow \infty} r_{n} / n=0,
$$

then (31) converges and there is no finite $\mu$ such that

$$
p(t) \leq e^{\mu t}
$$

for all $t$.

Proof. Let $f_{n}=\phi_{n}(u)$, and define a matrix $A=\left(a_{i j} ; i, j=1,2, \cdots\right)$ by

$$
a_{i 1}=f_{i}, \quad a_{i, i+1}=1 \quad(i=1,2, \ldots)
$$

with all other $a_{i j}$ zero. Then it is easy to check by induction on $n$ that the matrix powers $A^{n}=\left(a_{i j}^{(n)}\right)$ exist and that

$$
\begin{aligned}
a_{i j}^{(n)} & =\sum_{r=i}^{i-j+n} f_{r} u_{i-j+n-r} & & (j \leq n), \\
& =1 & & (j=n+i), \\
& =0 & & \text { (otherwise). }
\end{aligned}
$$


Then

$$
a_{11}^{(n)}=\sum_{r=1}^{n} f_{r} u_{n-r}=u_{n}
$$

and

$$
a_{i j}^{(n)} \leq \sum_{r=1}^{i-j+n} f_{r} u_{i-j+n-r}=u_{i-j+n} \quad(j \leq n) .
$$

Since (31) converges, so does

$$
p_{i j}(t)=\sum_{n=0}^{\infty} a_{i j}^{(n)} t^{n} e^{-t / n !}
$$

where $a_{i j}^{(0)}=\delta_{i j}$. Clearly the functions $p_{i j}$ satisfy (1) and (4), and since

$$
\frac{(s+t)^{n} e^{-(s+t)}}{n !}=\sum_{m=0}^{n} \frac{s^{m} e^{-s}}{m !} \cdot \frac{t^{n-m} e^{-t}}{(n-m) !}
$$

we have

$$
\begin{aligned}
p_{i j}(s+t) & =\sum_{m, r=0}^{\infty} a_{i j}^{(m+r)} \frac{s^{m} e^{-s}}{m !} \frac{t^{r} e^{-t}}{r !} \\
& =\sum_{m, r=0}^{\infty} \sum_{k=1}^{\infty} a_{i k}^{(m)} \frac{s^{m} e^{-s}}{m !} a_{k j}^{(r)} \frac{t^{r} e^{-t}}{r !} \\
& =\sum_{k=1}^{\infty} p_{i k}(s) p_{k j}(t),
\end{aligned}
$$

so that (3) also holds.

In particular, letting $u$ be the generalised sequence described in Proposition 3 , and assuming (33), we see that (31) converges since the ratio of successive terms is

$$
u_{n+1} \frac{t^{n+1} e^{-t}}{(n+1) !} / u_{n} \frac{t^{n} e^{-t}}{n !}=\frac{r_{n} t}{n+1}<1
$$

for large $n$ by (33). Moreover, if (34) were satisfied, we should have

$$
e^{\mu n} \geq p(n) \geq u_{n} n^{n} e^{-n} / n ! \geq u_{n} / 3 n
$$

implying the tameness of $u$, and contradicting (33). Thus the proof is complete.

If a family $\left(p_{i j}\right)$ satisfies (1), (3) and (4), if none of the $p_{i j}$ vanishes identically, and if $p_{11}(t) \leq e^{\mu t}$, then it is known [7] that there exist positive numbers $x_{j}$ such that 


$$
\sum_{j=1}^{\infty} p_{i j}(t) x_{j} \leq e^{\mu t} x_{i}
$$

for all $i, t$. Accordingly the functions

$$
\bar{p}_{i j}(t)=e^{-\mu t} p_{i j}(t) x_{j} / x_{i}
$$

satisfy (1), (2), (3) and (4), and the semigroup may then be brought within the ambit of the classical theory. The proposition asserts the existence of wild semigroups which cannot be so reduced, and for which more powerful methods, such as those of Jurkat, must be brought into play.

4. Semi-p-functions. A semi-p-function is defined to be a real-valued function $p$ of a positive real variable such that, for all $n \geq 1$ and $0<t_{1}<t_{2}<\cdots<t_{n}$, $p$ satisfies the inequality

$$
F\left(t_{1}, t_{2}, \cdots, t_{n} ; p\right) \geq 0
$$

where $F$ is defined by (6). More explicitly, if we write $t=t_{1}, u=t_{2}-t_{1}, v=$ $t_{3}-t_{2}, \cdots$, this inequality takes the form, for $n=1$,

$$
p(t) \geq 0
$$

for $n=2$,

$$
p(t+u)-p(t) p(u) \geq 0
$$

for $n=3$,

$$
p(t+u+v)-p(t) p(u+v)-p(t+u) p(v)+p(t) p(u) p(v) \geq 0
$$

and so on, for all $t, u, v, \cdots \geq 0$.

We shall in this paper only consider semi-p-functions which are standard in the sense that

$$
\lim _{t \rightarrow 0} p(t)=1
$$

Thus, unless otherwise stated, any semi- $p$-function will be supposed standard, and its definition will be extended from $(0, \infty)$ to $[0, \infty)$ by setting $p(0)=1$.

Theorem I. Every semi-p-function is strictly positive and continuous on $[0, \infty)$. The limit

$$
\Lambda=\lim _{t \rightarrow \infty} t^{-1} \log p(t)
$$

exists in $-\infty<\Lambda \leq \infty$. If $\Lambda$ is finite, then

$$
p(t) \leq e^{\mathbf{\Lambda} t}
$$

for all $t$, and the function 


$$
\bar{p}(t)=p(t) e^{-\Lambda t}
$$

is a p-function.

Proof. From (40), $p(t) \geq\{p(t / n)\}^{n}$, and (42) shows that the right-hand side is strictly positive if $n$ is taken large enough. Thus $p(t)>0$ for all $t \geq 0$.

For any $t>0$, choose two sequences $\left(\alpha_{m}\right),\left(\beta_{n}\right)$ converging to zero through positive values in such a way that

$$
\begin{aligned}
& \lim p\left(t+\alpha_{m}\right)=\lim _{u \rightarrow 0 ; u>0} \inf _{u>0} p(t+u), \\
& \lim p\left(t+\beta_{n}\right)=\limsup _{u \rightarrow 0 ; u>0} p(t+u) .
\end{aligned}
$$

Whenever $\alpha_{m}>\beta_{n}$, (40) shows that $p\left(t+\alpha_{m}\right) \geq p\left(t+\beta_{n}\right) p\left(\alpha_{m}-\beta_{n}\right)$, so that, using (42), $\lim p\left(t+\alpha_{m}\right) \geq \lim p\left(t+\beta_{n}\right)$. Hence $p(t+)=\lim _{u \rightarrow 0 ; u>0} p(t+u)$ exists, and similarly so does $p(t-)=\lim _{u \rightarrow 0 ; u>0} p(t-u)$; (40) shows that $p(t-) \leq p(t) \leq p(t+)(t>0)$. Notice that $p(0+)=p(0)=1$.

Since $p$ has left-and right-hand limits everywhere, its discontinuity set $D=\{t ; p(t-)<p(t+)\}$ is at most countable. In (41), let $v \rightarrow 0$ to give $p(t+u+)$ $-p(t+u) \geq p(t)\{p(u+)-p(u)\}$. Since $t$ can be chosen so that $t+u \notin D$, we have $p(u+)=p(u)$. Now write $s=u+v$, and let $v \rightarrow 0$ with $s$ fixed, to give $p(t+s)$ $-p(t+s-) \geq p(t)\{p(s)-p(s-)\}$ which shows that $p(s)=p(s-)$. Hence $D$ is empty and $p$ is continuous.

The function $\log p(t)$ is by (40) finite, continuous and superadditive. Hence, by Theorem 7.6.1 of [3], the limit (43) exists, and (44) holds if $\Lambda<\infty$.

Now suppose that $\Lambda$ is finite, and define $\bar{p}$ by (45). If, for $b>0, u^{b}$ denotes the sequence $u_{n}^{b}=\bar{p}(n b)$, then an easy computation (cf. [9]) show s that

$$
\phi_{n}\left(u^{b}\right)=F(b, 2 b, \cdots, n b ; \bar{p}) \geq 0,
$$

so that $u^{b}$ is a generalised renewal sequence. Since $u_{n}^{b} \leq 1, u^{b}$ is a renewal sequence, and since $\bar{p}$ is continuous, Proposition 6 of [9] implies that $\bar{p}$ is a $p$-function, and the theorem is proved.

A semi-p-function is called tame if $\Lambda$ is finite, and wild otherwise; the last part of the theorem shows that the study of tame semi-p-functions is an essentially trivial extension of that of $p$-functions. The existence of wild semi-p-functions is however guaranteed by Proposition 4, and the following result shows how other examples may be constructed.

Proposition 5. If the function $p$ bas the property that, for every $T>0$, there is a number $\lambda$ and a p-function $\tilde{p}$ such that

$$
p(t)=\tilde{p}(t) e^{\lambda t} \quad(0 \leq t \leq T),
$$

then $p$ is a semi-p-function. 
Proof. So long as $t_{n} \leq T, F\left(t_{1}, \cdots, t_{n} ; p\right)$ depends only on the values of $p$ on $[0, T]$, so that

$$
F\left(t_{1}, \cdots, t_{n} ; p\right)=e^{\lambda t} F\left(t_{1}, \cdots, t_{n} ; \tilde{p}\right) \geq 0 .
$$

Since $T$ is arbitrary, $p$ is a semi- $p$-function.

Proposition 6. Let $p$ be a positive continuous function on $[0, \infty)$ with $p(0)$ $=1$ such that $\log p$ is convex. Then $p$ is a semi-p-function.

Proof. Apply Proposition 5, with $\lambda=p^{\prime}(T) / p(T)$ and

$$
\begin{aligned}
\tilde{p}(t) & =p(t) e^{-\lambda t} & & (t \leq T), \\
& =p(T)^{-\lambda T} & & (t>T),
\end{aligned}
$$

noting that $\log \widetilde{p}$ is continuous, nonpositive and convex, and hence $\widetilde{p}$ is a $p$-function ([11], [6]).

5. The taming of wild semi-p-functions. The key to the study of wild semi$p$-functions is a converse to Proposition 5, a continuous-time version of Proposition 2, which asserts that a wild semi-p-function looks like a tame one on any finite interval. This is the only deep result of the paper.

Theorem II. If $p$ is a semi-p-function, and $0<T<\infty$, there exists a number $\lambda$ and a p-function $\tilde{p}$ such that

$$
p(t)=e^{\lambda t} \tilde{p}(t) \quad(0 \leq t \leq T) .
$$

Proof. The set

$$
E=\{t \geq 0 ; T p(t)=(t-T) p(2 T)\}
$$

is closed since $p$ is continuous, nonempty since $2 T \in E$, and lies within $(T, \infty)$. Let $S$ denote its greatest lower bound, so that $S \in E, T<S \leq 2 T$. Then the continuous function

$$
t \mapsto T p(t)-(t-T) p(2 T)
$$

does not vanish on $[0, S)$, and since it is positive on $[0, T]$, we must have

$$
T p(t)-(t-T) p(2 T)>0 \quad(0 \leq t<S) .
$$

Since $T p(S)-(S-T) p(2 T)=0$, we have $p(t) / p(S)>(t-T) /(s-T)$. From this it follows that the function

$$
t \mapsto(\log p(S)-\log p(t)) /(S-t),
$$

which is continuous on $[0, S)$, is also bounded above on $[\tau, S)$ if $T<\tau<S$. Hence we may choose $\lambda$ so large that 


$$
(\log p(S)-\log p(t)) /(S-t) \leq \lambda \quad(0 \leq t<S)
$$

and then

$$
p(S) e^{-\lambda S} \leq p(t) e^{-\lambda t} \quad(0 \leq t \leq S)
$$

For any positive integer $N$, define

$$
f_{n}=e^{-\lambda n b} F(b, 2 b, \cdots, n b ; p),
$$

where $b=S / N$. As in the proof of Theorem $I,\left(f_{n}\right)$ is the $\phi$-sequence of the generalised renewal sequence $e^{-\lambda n b} p(n b)$, so that $f_{n} \geq 0$. Define a generalised renewal sequence $u$ such that

$$
\begin{aligned}
\phi_{n}(u) & =f_{n} & & (1 \leq n \leq N), \\
& =0 & & (N<n) .
\end{aligned}
$$

Then

$$
u_{n}=e^{-\lambda n h} p(n b) \quad(0 \leq n \leq N)
$$

and (48) implies that $u_{N} \leq u_{n}(0 \leq n \leq N)$. Thus,

$$
u_{N}=\sum_{r=1}^{N} f_{r} u_{N-r} \geq \sum_{r=1}^{N} f_{r} u_{N}
$$

showing that $\sum_{r=1}^{N} f_{r} \leq 1$, and that $u$ is a renewal sequence.

Proposition 22 of [9] now implies that

$$
\widetilde{p}_{N}(t)=\sum_{n=0}^{\infty} u_{n}(t / h)^{n} e^{-t / b} / n !
$$

is a $p$-function $\tilde{p}_{N}$. If we combine (49), the continuity of $p$ on $[0, s]$, and the inequality $0 \leq u_{n} \leq 1$ with the well-known fact that

$$
\lim _{N \rightarrow \infty} \sum_{t-\eta<n b<t+\eta} \frac{(t / b)^{n} e^{-t / b}}{n !}=\sum_{n=0}^{\infty} \frac{(t / b)^{n} e^{-t / b}}{n !}=1
$$

for any $\eta>0$, it follows easily that

$$
\lim _{N \rightarrow \infty} \tilde{p}_{N}(t)=p(t) e^{-\lambda t} \quad(0 \leq t \leq S)
$$

Now recall that the (standard) $p$-functions, under the topology of uniform convergence on compact sets, form a metrisable space $\mathcal{P}$ in which the relatively compact subspaces are those which are equicontinuous at the origin [6]. In $\mathcal{P}$ the sequence $\left(\tilde{p}_{N}\right)$ is therefore relatively compact, and has a limit point $\tilde{p}$, which, by (51), satisfies $\tilde{p}(t)=p(t) e^{-\lambda t}$ on $0 \leq t \leq S$, and a fortiori on $0 \leq t \leq T$. Thus the theorem is proved.

An immediate corollary of the theorem is that the local analytical properties known to hold for $p$-functions are also true for semi-p-functions. Thus the theorems of [9] and [10] lead at once to the following result. 
Proposition 7. If $p$ is a semi-p-function, its right and left derivatives $D_{+} p(t)$ and $D_{\_} p(t)$ exist and are finite for all $t>0$, and satisfy

$$
D_{+} p(t) \geq D_{-} p(t)
$$

with equality for all but countably many values of $t$. In any interval throughout which equality bolds in (52), the derivative $p^{\prime}(t)$ is continuous. The right derivative at the origin exists, and

$$
-\infty \leq D_{+} p(0)<\infty, \quad D_{+} p(0) \leq \Lambda .
$$

For any $T>0$, let $L_{T}$ denote the set of values of $\lambda$ for which $p(t) e^{-\lambda t}$ coincides on $[0, T]$ with a $p$-function. By Theorem II, $L_{T}$ is nonempty, and it is bounded below since, for $\lambda \in L_{T}, p(T) e^{-\lambda T} \leq 1$. It is moreover closed, since for any $M$, the family of $p$-functions $p(t) e^{-\lambda t}\left(\lambda \in L_{T}, \lambda \leq M\right)$ is equicontinuous at $t=0$ and so relatively compact in $\mathcal{P}$. Hence $L_{T}$ attains its greatest lower bound $\Lambda(T)$. Clearly $L_{T} \supseteq L_{S}$ for $T<S$, so that $\Lambda(T)$ increases $w i$ ith $T$, and it is easy to see that

$$
\lim _{T \rightarrow \infty} \Lambda(T)=\Lambda
$$

where $\Lambda$ is defined by (43).

6. The Volterra equation. Theorem II clears the way for a systematic study of semi-p-functions along the lines of the existing theory of $p$-functions, but with one essential difference. It no longer makes sense to express results in terms of Laplace transforms, since the integral $\int_{0}^{\infty} p(t) e^{-\theta t} d t$ diverges for all $\theta$ if $p$ is a wild semi-p-function.

The way to avoid this difficulty is to rewrite (13) in the form

$$
\hat{p}(\theta)=\theta^{-1}\{1+\hat{m}(\theta)\}^{-1},
$$

where the function

$$
m(t)=\mu(t, \infty]
$$

is nonnegative, nonincreasing and right-continuous with $\int_{0}^{1} m(t) d t<\infty$. Then (55) is equivalent to $\hat{p}(\theta)+\hat{p}(\theta) \hat{m}(\theta)=\theta^{-1}$, and thus to the Volterra equation

$$
p(t)+\int_{0}^{t} p(t-s) m(s) d s=1 .
$$

The basic theorem is that, when we move from $p$-functions to semi- $p$-functions, the equation (57) still holds good, the only change being that the function $m$ need no longer be nonnegative. 
Theorem III. If $p$ is a semi-p-function, there exists a unique nonincreasing right-continuous function $m$ on $(0, \infty)$, integrable over $(0,1)$, such that $(57)$ bolds for all $t>0$. Conversely, if $m$ is any such function, there is a unique continuous function $p$ satisfying (57), and $p$ is a semi-p-function. It is a p-function if and only if $m(t) \geq 0$ for all $t>0$.

Proof. Let $p$ be a semi-p-function, and define $\Lambda(T)$ as at the end of $\S$, so that $p(t)=e^{\boldsymbol{\Lambda}(T) t} p_{T}(t)(0 \leq t \leq T)$ for some $p$-function $p_{T}$. Define $m_{T}(t)=$ $\mu_{T}(t, \infty]$, where $\mu_{T}$ is the canonical measure of $p_{T}$, and write

$$
m^{T}(t)=-\Lambda(T)+m_{T}(t) e^{\Lambda(T) t}-\Lambda(T) \int_{0}^{t} m_{T}(s) e^{\Lambda(T) s} d s .
$$

Do the same thing for some $U<T$; write $p(t)=e^{\mathbf{\Lambda}(U) t} p_{U}(t)(0 \leq t \leq U)$ and define $m_{U}$ and $m^{U}$. Then

$$
p_{T}(t)=e^{-\lambda t} p_{U}(t) \quad(0 \leq t \leq U),
$$

where $\lambda=\Lambda(T)-\Lambda(U)$. Now it is known [10], and evident from the form of the integral equation (57), that the values of $m$ on $(0, U)$ determine those of $p$ on $(0, U)$, and vice versa. Therefore $m_{T}$ coincides on $(0, U)$ with the $m$-function $m_{U, \lambda}$ of the $p$-function $p_{U}(t) e^{-\lambda t}$. But

and

$$
\begin{aligned}
\theta^{-1}\left\{1+\hat{m}_{U, \lambda}(\theta)\right\}^{-1} & =\int_{0}^{\infty} p_{U}(t) e^{-\lambda t} e^{-\theta t} d t \\
& =\hat{p}(\theta+\lambda)=(\theta+\lambda)^{-1}\left\{1+\hat{m}_{U}(\theta+\lambda)\right\}^{-1},
\end{aligned}
$$

$$
m_{U, \lambda}(t)=\lambda+m_{U}(t) e^{-\lambda t}+\lambda \int_{0}^{t} m_{U}(s) e^{-\lambda s} d s .
$$

Hence, for $0<t<U$,

$$
m_{T}(t)=\lambda+m_{U}(t) e^{-\lambda t}+\lambda \int_{0}^{t} m_{U}(s) e^{-\lambda s} d s,
$$

and substituting this into (58) and simplifying gives

$$
m^{T}(t)=m^{U}(t) \quad(0<t<U) .
$$

It follows from $(60)$ that there is a function $m$ defined on $(0, \infty)$ such that, for every $T>0$,

$$
m^{T}(t)=m(t) \quad(0<t<T)
$$

If $(58)$ is integrated by parts, we have

$$
m^{T}(t)=m^{T}(T)-\int_{(t, T]} e^{\Lambda(T) s} \mu_{T}(d s),
$$

which shows that $m^{T}$ is nonincreasing and right-continuous in $(0, T)$. Hence $m$ is nonincreasing and right-continuous in $(0, \infty)$. 
Another consequence of (58) is that the Laplace transform of $m^{T}$ exists in $\theta>\max \{\Lambda(T), 0\}$, and that

$$
\begin{aligned}
\hat{m}^{T}(\theta) & =-\Lambda(T) \theta^{-1}+\hat{m}_{T}(\theta-\Lambda(T))-\Lambda(T) \theta^{-1} \hat{m}_{T}(\theta-\Lambda(T)) \\
& =\left\{1-\Lambda(T) \theta^{-1}\right\}\{1+\hat{m}(\theta-\Lambda(T))\}-1 \\
& =\theta^{-1} \hat{p}_{T}(\theta-\Lambda(T))^{-1}-1
\end{aligned}
$$

so that

$$
\hat{p}_{T}(\theta-\Lambda(T))\left\{1+\hat{m}^{T}(\theta)\right\}=\theta^{-1} \text {. }
$$

This implies that

$$
p_{T}(t) e^{\Lambda(T) t}+\int_{0}^{t} p_{T}(t-s) e^{\Lambda(T)(t-s)} m^{T}(s) d s=1,
$$

so that

$$
p(t)+\int_{0}^{t} p(t-s) m^{T}(s) d s=1 \quad(0 \leq t \leq T) .
$$

By (61) this means that $p$ and $m$ satisfy (57).

Conversely, suppose that $m$ is a nonincreasing right-continuous function on $(0, \infty)$, integrable on $(0,1)$. For $T>0$, define

$$
\tilde{m}(t)=\lambda+m(t) e^{-\lambda t}+\lambda \int_{0}^{t} m(s) e^{-\lambda s} d s \quad(0<t<T),
$$

where $\lambda$ is chosen so large that $\widetilde{m}(T) \geq 0$. Then as before $\tilde{m}$ is nonincreasing and right-continuous, and there is a measure $\tilde{\mu}$ on $(0, \infty]$ such that

$$
\begin{array}{rlrl}
\tilde{\mu}(t, \infty] & =\tilde{m}(t) & & (0<t<T), \\
& =\tilde{m}(T) \quad(T \leq t) ;
\end{array}
$$

let $\tilde{p}$ be the $p$-function with canonical measure $\tilde{\mu}$, and $p^{(T)}$ the semi-p-function $p^{(T)}(t)=e^{\lambda t} \tilde{p}(t)$. Computations just like those already described show that, if $U<T$, then

$$
p^{(T)}(t)=p^{(U)}(t) \quad(0 \leq t \leq U) .
$$

Hence there is a function $p$ which, for any $T>0$, coincides on $[0, T]$ with the semi- $p$-function $p^{(T)}$, and Proposition 5 shows that $p$ is a semi-p-function. Hence the theorem is proved.

Theorem III characterises the (standard) semi-p-functions by setting up a oneto-one correspondence, expressed by (57), between them and the locally integrable nonincreasing functions $m$. It might perhaps be more logical to write $l=-m$, so that (57) becomes

$$
p=1+p * l
$$


except that in the crucial special case of $p$-functions $l$ would then always be negative.

Just as in that special case, the equation (57) has useful consequences which will not be proved here. For instance, in the obvious iterative solution of (57),

$$
p(t)=1+\int_{0}^{t} \sum_{n=1}^{\infty}(-1)^{n} m_{n}(s) d s,
$$

where $m_{n}$ is the $n$-fold convolution of $m$ with itself, the series is absolutely convergent, uniformly in every compact subset of $(0, \infty)$. As $t \rightarrow 0$,

$$
1-p(t) \sim \int_{0}^{t} m(s) d s
$$

and, in particular,

$$
D_{+} p(0)=-m(0)
$$

7. The extrapolation of semi-p-functions. Theorem II asserts that, if a function is defined on $[0, T]$ and is known to be the restriction of some semi- $p$-function, then it may be extrapolated to $(0, \infty)$ in such a way that the extrapolated function is a tame semi-p-function. This result leaves open two questions:

(1) How can we tell whether a function on $[0, T]$ is the restriction of some semi-p-function?

(2) The extrapolation evidently being nonunique, is there some canonical extrapolation having some special property?

Proposition 8. Let $p$ be a continuous function on $[0, T]$ with $p(0)=1$. Then $p$ is the restriction of some semi-p-function if and only if, whenever $n \geq 1$ and $0<t_{1}<t_{2}<\cdots<t_{n} \leq T$,

$$
F\left(t_{1}, t_{2}, \cdots, t_{n} ; p\right) \geq 0
$$

Proof. The necessity of the condition is evident. To prove the sufficiency, we sharpen the arguments used to prove Theorem II. For any $\tau<T$, the set

$$
\{t ; 0 \leq t \leq T,(T-\tau) p(t)=(t-\tau) p(T)\}
$$

is nonempty since it contains $T$, closed since $p$ is continuous, and lies wholly within $(\tau, T]$. Hence its greatest lower bound $S$ satisfies

$$
\tau<S \leq T, \quad(T-\tau) p(S)=(S-\tau) p(T) .
$$

The function $t \mapsto(T-\tau) p(t)-(t-\tau) p(T)$ must therefore be of constant sign on 
$[0, s)$, and this shows, as before, that $\lambda$ can be chosen so that

$$
p(S) e^{-\lambda S} \leq p(t) e^{-\lambda t} \quad(0 \leq t \leq S) .
$$

Now define, for any $N \geq 1$, a sequence $a=\left(a_{n}\right)$ by

$$
\begin{aligned}
a_{n} & =p(n S / N) e^{-\lambda n S / N} & & (n \leq N), \\
& =0 & & (n>N) .
\end{aligned}
$$

Then $a$ is not a renewal sequence, but, for $n \leq N$,

$$
f_{n}=\phi_{n}(a)=F(S / N, 2 S / N, \ldots, n S / N ; p) e^{-\lambda S / N} \geq 0
$$

by (67). As before (68) shows that $\sum_{n=1}^{N} f_{n} \leq 1$, so that there is a renewal sequence $u$ with

and

$$
\begin{aligned}
\phi_{n}(u) & =f_{n} & & (n \leq N), \\
& =0 & & (n>N),
\end{aligned}
$$

$$
u_{n}=a_{n} \quad(n \leq N) .
$$

Then (50) defines a $p$-function $\widetilde{p}_{N}$ which converges, as $N \rightarrow \infty$, to $p(t) e^{-\lambda t}$ on $[0, s]$. Hence the latter function is the restriction to $[0, s]$ of a $p$-function $\tilde{p}$.

Since $S>\tau$, we have therefore proved that, for any $\tau<T$, there is a semi$p$-function $p_{\tau}$ such that

$$
p_{\tau}(t)=p(t) \quad(0 \leq t \leq \tau) .
$$

Let $m_{\tau}$ be the function corresponding in Theorem III to $p_{\tau}$. Then, for $\sigma<\tau$, since $p_{\sigma}(t)=p_{\tau}(t) \quad(0 \leq t \leq \sigma)$, we have $m_{\sigma}(t)=m_{\tau}(t) \quad(0<t<\sigma)$. Hence there is a nonincreasing right-continuous function $m$ on $(0, T)$ such that, for all $\tau<T$,

$$
m_{\tau}(t)=m(t) \quad(0<t<\tau) .
$$

Extend the definition of $m$ to $(0, \infty)$ by setting $m(t)=m(T-)(t \geq T)$, and use Theorem III to construct the corresponding semi-p-function $\bar{p}$. By (70) and (69), $\bar{p}(t)=p_{\tau}(t)=p(t) \quad(0 \leq t \leq \tau)$, and since $\tau<T$ is arbitrary, and $p$ and $\bar{p}$ continuous, we deduce that $\bar{p}(t)=p(t) \quad(0 \leq t \leq T)$, which completes the proof.

Turning now to the second question, if a function $p$ on $[0, T]$ is known to be the restriction of some semi-p-function, then $p$ determines the function $m$ on $(0, T)$. The extrapolation of $p$ is therefore equivalent to the extrapolation of $m$ from $(0, T)$ to $(0, \infty)$, and this can be done in any way which respects the nonincreasing and right-continuous nature of $m$. A special position is clearly occupied by the extrapolation which makes $m$ constant on $[T, \infty)$;

$$
m(t)=m(T-) \quad(T \leq t) .
$$


These considerations also make it possible to evaluate the quantity $\Lambda(T)$. The semi-p-function $p(t) e^{-\lambda t}$ has $m$-function

$$
m_{\lambda}(t)=\lambda+m(t) e^{-\lambda t}+\lambda \int_{0}^{t} m(s) e^{-\lambda s} d s .
$$

Thus $\lambda \geq \Lambda(T)$ if and only if there is a nonnegative nonincreasing function coinciding with $m_{\lambda}$ on $(0, T)$, and this will occur if and only if $m_{\lambda}(T) \geq 0$. Hence $\Lambda(T)$ is the smallest value of $\lambda$ for which

$$
\lambda+m(T) e^{-\lambda T}+\lambda \int_{0}^{T} m(s) e^{-\lambda s} d s \geq 0 .
$$

In principle, this is determined by the values of $p$ on $[0, T]$, but in practice the calculation would rarely be feasible.

\section{REFERENCES}

1. K. L. Chung, Markov chains with stationary transition probabilities, Die Grundlehren der math. Wissenschaften, Band 104, Springer-Verlag, Berlin and New York, 1960. MR 22 \#176.

2. A. G. Cornish, Some properties of semigroups of non-negative matrices and their resolvents, Proc. London Math. Soc. 23 (1971), 643-667.

3. E. Hille and R. S. Phillips, Functional analysis and semi-groups, rev. ed., Amer. Math. Soc. Colloq. Publ., vol. 31, Amer. Math. Soc., Providence, R.I., 1957. MR 19, 664.

4. W. B. Jurkat, On semi-groups of positive matrices. I, II, Scripta Math. 24 (1959), 123-131, 207-218. MR $22 \# 12563$.

5. - On the analytical structure of semi-groups of positive matrices, Math. Z. 73 (1960), 346-365. MR 23 \#A524.

6. D. G. Kendall, Delphic semi-groups, infinitely divisible regenerative phenomena, and the arithmetic of p-functions, Z. Wahrscheinlichkeitstheorie und Verw. Gebiete 9 (1968), 163-195. MR 37 \#5320.

7. J. F. C. Kingman, The exponential decay of Markov transition probabilities, Proc. London Math. Soc. (3) 13 (1963), 337-358. MR 27 \#1995.

8. - A continuous time analogue of the theory of recurrent events, Bull. Amer. Math. Soc. 69 (1963), 268-272. MR $26 \# 7044$.

9 . - - The stochastic theory of regenerative events, Z. Wahrscheinlichkeitstheorie und Verw. Gebiete 2 (1964), 180-224. MR $32 \# 1758$.

10. - Some further analytical results in the theory of regenerative events, J. Math. Anal. Appl. 11 (1965), 422-433. MR 31 \#5237.

11. - An approach to the study of Markov processes (with discussion), J. Roy. Statist. Soc. Ser. B 28 (1966), 417-447. MR 35 \#1085.

12. Markov transition probabilities. V, Z. Wahrscheinlichkeitstheorie und Verw. Gebiete 17 (1971), 89-103.

13. (1) - Regenerative phenomena, Wiley, London, 1972.

14. A. N. Kolmogorov, On the differentiability of the transition probabilities in stationary Markov processes with a denumerable number of states, Uc'. Zap. Moskov. Gos. Univ. 148 Mat. 4(1951), 53-59. (Russian) MR 14, 295.

MATHEMATICAL INSTITUTE, UNIVERSITY OF OXFORD, OXFORD OX1 3LB, ENGLAND

(1) All the results for which the reader has been referred to [3], [6], [8], [9], [10], $[11]$ or [12] may also be found in this reference. 